\title{
Risk factors for hypothyroidism in euthyroid thy- roid nodule patients with lymphocytic thyroiditis on fine needle aspiration cytology
}

Jeong-Min Lee ${ }^{1}$, Jeonghoon $\mathrm{Ha}^{1}$, Kwanhoon $\mathrm{Jo}^{1}$, Yejee $\mathrm{Lim}^{1}$, Min-Hee Kim ${ }^{1}$, Chan-Kwan Jung ${ }^{2}$, So-Lyung Jung ${ }^{3}$, Moo-Il Kang ${ }^{1}$, Bong-Yun $\mathrm{Cha}^{1}$, and Dong-Jun Lim ${ }^{1}$

${ }^{1}$ Division of Endocrinology and Metabolism, Department of Internal Medicine, Departments of ${ }^{2}$ Pathology and ${ }^{3}$ Radiology, College of Medicine, Seoul St. Mary's Hospital, The Catholic University of Korea, Seoul, Korea

\author{
Received: May 13, 2017 \\ Revised : September 16, 2017 \\ Accepted: October 29, 2017

\section{Correspondence to} \\ Dong-Jun Lim, M.D. \\ Division of Endocrinology and \\ Metabolism, Department of Inter- \\ nal Medicine, College of Medicine, \\ Seoul St. Mary's Hospital, The \\ Catholic University of Korea, 222 \\ Banpo-daero, Seocho-gu, Seoul \\ 06591, Korea \\ Tel: $+82-2-2258-6009$ \\ Fax: +82-2-599-3589 \\ E-mail:1dj6026@catholic.ac.kr
}

Background/Aims: Lymphocytic thyroiditis as cytology diagnosis from fine needle aspiration (FNA) is frequently detected in patients with thyroid nodules. However, the clinical outcome for upcoming hypothyroid events has been rarely clarified in euthyroid patients.

Methods: We retrospectively reviewed the data of patient who had lymphocytic thyroitidis on FNA cytology of thyroid nodule from January 2005 to December 2010 at a tertiary referral hospital. In total, 109 patients with follow-up thyroid function tests (TFT) were enrolled. Final outcomes included overt and subclinical hypothyroidism with thyroid stimulating hormone (TSH) levels $\geq 10 \mathrm{mIU} / \mathrm{L}$. Potential parameters predicting clinical hypothyroidism were analyzed by multivariate analysis.

Results: Over the mean follow-up duration of 51.6 months, 14 out of 109 patients (12.8\%) developed clinical hypothyroidism that required thyroid hormone replacement. The median onset time to hypothyroidism was 16 months (range, 3 to 88 ) and $\geq 60 \%$ of patients experienced clinical hypothyroidism within 1 year. By multivariate analysis, background thyroiditis (relative risk [RR], 9.78; $p=0.004$ ), thyroid peroxidase antibody positivity ( $R R, 9.90 ; p=0.003)$, nodule size $(R R, 1.24$; $p<0.001)$, and initial TSH $(\mathrm{RR}, 1.47 ; p=0.009)$ were the independent risk factors for predicting hypothyroidism in euthyroid patients.

Conclusions: Hypothyroidism frequently occurs during the follow-up in euthyroid patients with thyroid nodules which show lymphocytic thyroiditis on FNA cytology. Close surveillance and regular TFT are needed in high-risk patients for upcoming clinical hypothyroidism.

Keywords: Thyroid nodule; Lymphocytic thyroiditis; Clinical hypothyroidism; Thyroid function tests; Biopsy, fine-needle

\section{INTRODUCTION}

Thyroid nodules are common and estimated prevalence of thyroid nodules is $5 \%$ to $7 \%$ with clinical palpitation and $30 \%$ to $50 \%$ with thyroid ultrasonography (US) [1].
Approximately $90 \%$ to $95 \%$ of the thyroid nodules are benign, although $5 \%$ are malignant [2]. Fine-needle aspiration cytology (FNAC) is considered the best tool for differentiating between these forms [3]. Previous clinical guidelines have recommended ultrasonographic (US) 
observation and follow-up for nodules that were identified as benign based on FNAC. Lymphocytic thyroiditis is the second most common thyroid lesion to be diagnosed using this technique [4]. Patients with evidence of thyroiditis based on the presence of thyroid autoantibody or US findings eventually develop subclinical or overt hypothyroidism. Therefore, lymphocytic thyroiditis in patients with normal thyroid stimulating hormone (TSH) levels requires careful follow-up to identify for the development of clinical hypothyroidism [5].

However, there is no consensus regarding follow-up using thyroid function testing (TFT) when a patient exhibits a euthyroid status and the thyroid nodules exhibit lymphocytic thyroiditis on FNAC. A few reports have indicated that some patients exhibit hypothyroidism during follow-up to confirm that FNAC diagnosis of thyroiditis, although it is difficult to determine the required follow-up tools for cases with lymphocytic thyroiditis. Therefore, the present study aimed to determine the appropriate follow-up tools in these cases, and to identify the risk factors for progression to clinical hypothyroidism.

\section{METHODS}

\section{Study subjects}

We retrospectively reviewed data from patients who underwent US-guided FNAC for thyroid nodules from January 2005 to December 2010 at a tertiary referral hospital. This search identified 289 individuals with a cytological diagnosis of lymphocytic thyroiditis. Exclusion criteria were (1) current thyroid hormone replacement therapy ( $\mathrm{n}=62)$, (2) absence of an initial TFT ( $\mathrm{n}=37)$, and (3) no regular visit with follow-up of TFT or thyroid US $(n=81)$. Thus, we initially included 109 patients in the present study.

During follow-up period, seven patients among 109 were diagnosed with papillary thyroid cancer and were excluded from the study due to the difficulty in differentiation between true Hashimoto's thyroiditis and peritumoral lymphocyte infiltration around papillary thyroid carcinoma.

\section{Follow-up TFT and thyroid US}

The follow-up interval of thyroid US was 12 months.
However, the follow-up interval of TFT varied from 3 to 6 months. If there was a significant change in the TFT, follow-up was performed within 3 months at the discretion of the clinician. Thus, the follow-up intervals were 6 to 12 month, and the patients underwent TFT and thyroid US at least three times.

\section{Definition of thyroid status and clinical outcomes according to TFT}

The TFT was performed. Also, thyroid peroxidase antibody (TPOAb) and thyroglobulin antibody (TgAb) were measured by radioimmunoassay. Our institution's uses the following reference ranges TSH 0.17 to $4.05 \mathrm{mIU} / \mathrm{mL}$, free thyroxine ( $\left.\mathrm{T}_{4}\right) 0.85$ to $1.86 \mathrm{ng} / \mathrm{dL}$, triiodothyronine (T3) 0.78 to $1.82 \mathrm{ng} / \mathrm{mL}$, TPOAb o to $80 \mathrm{IU} / \mathrm{mL}$, and $\mathrm{TgAb}$ o to $70 \mathrm{IU} / \mathrm{mL}$. Patients were assigned to group E (euthyroidism) if they had normal values for serum TSH and free $\mathrm{T}_{4}$ [6]. Subclinical hypothyroidism was defined as a serum TSH concentration that was above the upper limit of the reference range, but with normal free $\mathrm{T}_{4}$ levels [5]. Overt hypothyroidism was generally defined as serum TSH concentrations above the reference range and free $\mathrm{T}_{4}$ levels below the reference range. However, some individuals with clinical symptoms of hypothyroidism, high TSH levels (> $10 \mathrm{mIU} / \mathrm{L}$ ), and low but normal free $\mathrm{T}_{4}$ levels were classified as having overt hypothyroidism [7]. High initial TSH levels and TPOAb positivity are risk factors for overt hypothyroidism. Therefore, in this study, the clinical hypothyroidism group ( $\mathrm{H}$ group) consisted of patients with both overt and subclinical hypothyroidism with TSH levels $\geq 10 \mathrm{mIU} / \mathrm{L}$. Clinical hypothyroidism was confirmed on repeat TFT at minimum 2 months after that initial assessment. After repeat TFT, if a patient presented high TSH and free $\mathrm{T}_{4}$ levels within the reference range, the clinician made decision according to patients' clinical presentation.

\section{Cytological diagnosis}

The FNAC was performed using a $22 \mathrm{G}$ Franseen needle under US guidance and the specimens were smeared onto glass slides and placed in 95\% alcohol for Papanicolaou staining. The classical cytological features of lymphocytic thyroiditis were accompanied by some plasma cells and macrophages. The lymphocytes have close contact with the thyrocytes and mediate their destruction. The cytological findings of lymphocytic thyroiditis 
are a mixed group of follicular and Hurthle cells with low-to-moderate colloid and lymphocytic infiltration in the epithelium $[8,9]$. A pathologist assessed the Hurthle cell changes, as well as the increased numbers of mature and transformed lymphocytes impinging on follicular cells [8]. Lymphocytic thyroiditis was diagnosed when the FNAC revealed a mixture of follicular cells and Hurthle cells in a background of mature lymphocytes (Supplementary Fig. 1). Two pathologists interpreted the cytological findings.

\section{Thyroid US}

The US findings for thyroid nodules that were evaluated using FNAC were also examined to determine their echogenicity, margin, shape, homogeneity, calcification, and size, based on previously reported methods [10]. Size was defined based on the maximum nodule diameter. Nodules with confirmed lymphocytic thyroiditis based on FNAC were categorized according to the grading system that was suggested by Sostre and Reyes [11], with grade 1 assigned to an enlarged gland with a normoechoic pattern, grade 2 nodules exhibiting multiple hypoechoic foci or patches scattered throughout the normoechoic gland (i.e., focal rather than diffuse involvement), grade 3 defined as an enlarged gland with diffuse but mild hypoechogenicity, and grade 4 nodules exhibiting marked hypoechogenicity (Fig. 1) [12,13].

\section{Statistical analysis}

All statistical analyses were performed using SPSS software version 14 (SPSS Inc., Chicago, IL, USA). The distributions of all continuous variables were examined using the Shapiro-Wilk test. The non-normally distributed variables were follow-up duration and TFT results (levels of TSH, free $\mathrm{T}_{4}$, and $\mathrm{T}_{3}$ ), which were reported as median and interquartile range. Normally distributed continuous variables were reported as mean \pm standard deviation. Number and percentage were used for all categorical variables (sex, background thyroiditis, TPOAb positivity, and $\mathrm{TgAb}$ positivity). The characteristics of groups $\mathrm{E}$ and $\mathrm{H}$ were compared using the independent $t$ test (continuous variables) or the chi-square and Fisher exact test (categorical variables). The outcomes for groups $\mathrm{E}$ and $\mathrm{H}$ were evaluated without adjusting for the time until the detection of hypothyroidism, and times to events were calculated based on the start of levothyrox-

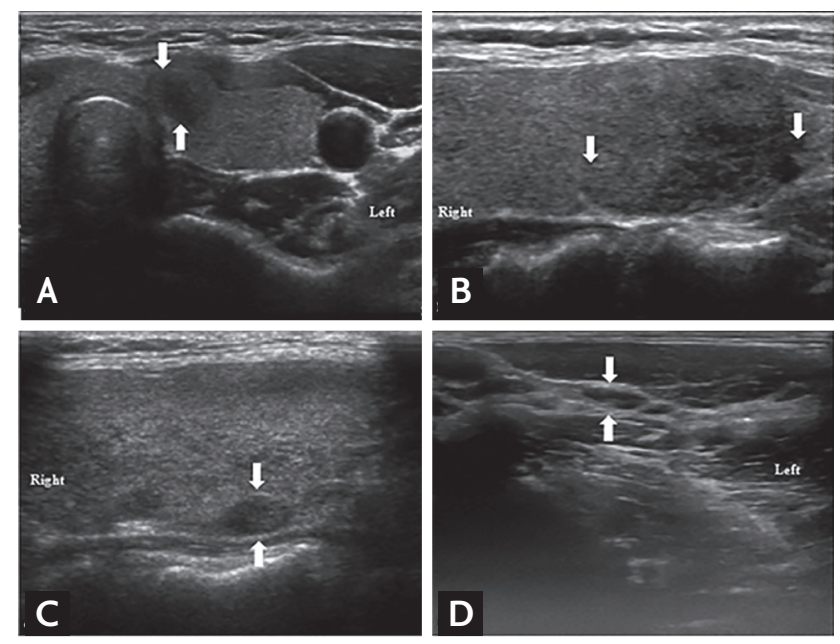

Figure 1. Grading background thyroiditis using thyroid ultrasonography. Grading based on ultrasonographic imaging was performed according to the suggestions of Sostre and Reyes [11]. (A) Grade 1: a 34-year-old woman with a 0.79-cm hypoechoic nodule (arrows) on the left isoechoic thyroid gland. (B) Grade 2: an 87-year-old woman with multiple hypoechoic foci through the right thyroid. (C) Grade 3: a 53-year-old woman with a 1.5-cm hypoechogenic nodule (arrows) and moderate coarse echogenicity. (D) Grade 4: a 57-year-old woman with a 1.1-cm hypoechoic ovoid nodule (arrows) on her very coarse and markedly hypoechogenic left thyroid gland.

ine therapy. Cox proportional hazards regression analysis was adjusted for potential confounders $(p<0.05$ in the univariate analyses) and used to evaluate the associations for the TFT and thyroid US findings. The results were reported as the relative risk (RR), $95 \%$ confidence interval (CI), and corresponding $p$ value. The cut-off values for nodule size $(17 \mathrm{~mm})$ and TSH levels (2.02 $\mathrm{mIU} / \mathrm{L})$ were determined using receiver operating characteristic curve analysis. Differences were considered statistically significant at a two-tailed $p$ value of $<0.05$.

\section{Ethical statements}

This study complied with the ethical standards of the Helsinki Declaration and was approved by the Catholic University of Korea, Catholic Medical Center, Seoul St, Mary's Hospital Institutional Review Board (IRB approval No. KCT18RESIo831).

This study was exempted from the consent of the IRB, because there is no reason to estimate the refusal of consent of the subject and the risk to the subject is extremely low even if the consent is waived. 
Table 1. Baseline clinical characteristics of study subjects and their thyroid function test at the time of fine needle aspiration $(n=109)$

\begin{tabular}{|c|c|}
\hline Characteristic & Value \\
\hline Age, yr & $49 \cdot 3 \pm 11.9$ \\
\hline Sex, women/men & $98 / 11$ \\
\hline Follow-up duration, mon & $58.7(23-94)$ \\
\hline TFT follow-up interval, mon & $10.2(3-18)$ \\
\hline No. of nodule & $1.6 \pm 0.8$ \\
\hline Nodule size, mm & $17.9 \pm 8.8$ \\
\hline \multicolumn{2}{|l|}{ Nodule characteristics $(\mathrm{n}=178)$} \\
\hline Hypoechoic nodule & $126(70.8)$ \\
\hline Nonparallel & $58(32.6)$ \\
\hline Calcification & $30(16.9)$ \\
\hline Microcalcification & $22 / 30$ \\
\hline Macrocalcification & $8 / 30$ \\
\hline \multicolumn{2}{|l|}{ Background thyroiditis on US } \\
\hline Isoechoic $(\mathrm{Gl})$ & $56(51.4)$ \\
\hline Multiple hypoenchoic foci $(\mathrm{G} 2)$ & $26(23.9)$ \\
\hline Mild hypoechoic $\left(\mathrm{G}_{3}\right)$ & $25(22.9)$ \\
\hline Marked hypoechoic (G4) & $2(1.8)$ \\
\hline TSH, mIU/L (0.17-4.05) & $2.20 \pm 1.33$ \\
\hline Free $\mathrm{T}_{4}, \mathrm{ng} / \mathrm{dL}(0.85-1.86)$ & $1.14 \pm 0.67$ \\
\hline $\mathrm{T}_{3}, \mathrm{ng} / \mathrm{mL}(0.78-1.82)$ & $1.04 \pm 0.59$ \\
\hline TPOAb positivity & $51(46.8)$ \\
\hline TgAb positivity & $43(39.4)$ \\
\hline
\end{tabular}

Values are presented as mean $\pm \mathrm{SD}$, median (range), or number (\%).

TFT, thyroid function test; US, ultrasonography; TSH, thyroid stimulating hormone; $\mathrm{T}_{4}$, thyroxine; $\mathrm{T}_{3}$, triiodothyronine; $\mathrm{TPOAb}$, thyroid peroxidase antibody; $\mathrm{TgAb}$, thyroglobulin antibody.

\section{RESULTS}

\section{Baseline clinical characteristics}

The patients' baseline clinical characteristics and TFT findings are shown in Table 1 . The median follow-up interval was 10.2 months (range, 3 to 18 ). The median number of nodules observed on thyroid US was 1.0 (range, 1.0 to 2.0 ) and the mean nodule size as $17.9 \mathrm{~mm}$ (range, 9.1 to 26.7). Thyroid US findings for nodules that were evaluated by FNAC were also analyzed again according to echogenicity, calcification, margin, and shape. Hy- poechogenicity was detected for 126 of the 178 nodules (70.8\%), and 30 of the nodules (16.9\%) were accompanied by calcification. Lobulated and poorly defined margins were observed for 71 nodules (39.9\%). The orientation was non-parallel (taller than wide) for 58 nodules (32.6\%). According to the Korean Thyroid Imaging Reporting and Data System (K-TIRADS) [14], 35 of the 178 nodules (19.7\%) were considered highly suspicious (K-TIRADS 5), 82 nodules $(46.1 \%)$ had an intermediate suspicion (K-TIRADS 4), 47 nodules (26.4\%) had a low suspicion (K-TIRADS 3), and 14 nodules (7.9\%) were considered benign (K-TIRADS 2). No significant differences were observed in the nodule characteristics when we compared groups $\mathrm{E}$ and $\mathrm{H}$ (data not shown).

The US findings of background thyroiditis were classified as grade 1 to 2 for 82 of the 109 patients (75.3\%) and as grade 3 to 4 for 27 patients (24.8\%). Fifty-one patients (46.8\%) were positive for TPOAb and 43 patients (39.4\%) were positive for $\mathrm{TgAb}$. Table 2 shows the clinical characteristics and TFT findings for groups $\mathrm{E}$ and $\mathrm{H}$. No significant intergroup differences were observed for age, sex, follow-up duration, number of nodules, and initial TFT findings (levels of TSH, free $\mathrm{T}_{4}$, and $\mathrm{T}_{3}$ ). However, group $\mathrm{H}$ had significantly larger nodules, compared to group E (27.5 mm vs. $15.1 \mathrm{~mm}, p<0.001)$. Grade 3 to 4 background thyroiditis was observed for 10 of the 14 patients in group $\mathrm{H}(71.4 \%)$ and in 10 of the 88 patients in group $\mathrm{E}$ (11.4\%). Group $\mathrm{H}$ had a higher proportion of TPOAb positivity, compared to group E (78.5\% vs. $43.2 \%$, $p<0.001)$. The difference in $\mathrm{TgAb}$ positivity was not statistically significant $(57.1 \%$ vs. $25 \%, p=0.068)$.

\section{Clinical outcome for lymphocytic thyroiditis}

We evaluated the outcomes of 109 patients with lymphocytic thyroiditis based on FNAC. Eighty-eight patients did not exhibit any symptoms or TFT abnormalities until the last follow-up visit. However, 14 of the 109 patients (12.8\%) progressed to clinical hypothyroidism during a mean follow-up of 58.7 months (range, 23 to 94). The detailed characteristics of these cases are shown in Table 3. Their median time to the onset of hypothyroidism was 16 months (range, 3 to 88 ), and nine of the 14 patients (64.3\%) developed clinical hypothyroidism within 1 year. Eleven patients exhibited progression to overt hypothyroidism and received levothyroxine therapy $(25$ to $75 \mu \mathrm{g})$. The remaining three patients underwent 
Table 2. Association between clinical characteristics and development hypothyroidism during follow-up

\begin{tabular}{|c|c|c|c|}
\hline Characteristic & $\begin{array}{l}\text { Euthyroid } \\
\qquad(\mathrm{n}=88)\end{array}$ & $\begin{array}{l}\text { Hypothyroidism }{ }^{\mathrm{a}} \\
(\mathrm{n}=14)\end{array}$ & $p$ value \\
\hline Age, yr & $50.4 \pm 11.9$ & $47.6 \pm 12.4$ & 0.521 \\
\hline Female sex & $80 / 88(90.9)$ & $12 / 14(85 \cdot 7)$ & 0.544 \\
\hline Follow-up duration, mon & $48.9 \pm 32.3$ & $58.7 \pm 35.1$ & 0.219 \\
\hline TFT follow-up interval, mon & $12.3 \pm 8.4$ & $7 \cdot 3 \pm 2.8$ & $<0.001$ \\
\hline No. of nodule & $1.5 \pm 0.68$ & $2.0 \pm 1.0$ & 0.093 \\
\hline Nodule size, mm & $15.10 \pm 5 \cdot 31$ & $27.52 \pm 11.65$ & $<0.001$ \\
\hline Background thyroiditis on US $\left(\mathrm{G}_{1}-\mathrm{G}_{2} / \mathrm{G}_{3}-\mathrm{G}_{4}\right)$ & $78 / 10$ & $4 / 10$ & $<0.001$ \\
\hline Onset of hypothyroidism (period) & & $16(3-88)$ & \\
\hline \multicolumn{4}{|l|}{ Initial thyroid function tests } \\
\hline TSH, mIU/L & $0.92 \pm 0.48$ & $1.03 \pm 0.43$ & 0.073 \\
\hline Free $\mathrm{T}_{4}, \mathrm{ng} / \mathrm{dL}(0.85-1.86)$ & $1.10 \pm 0.46$ & $1.18 \pm 0.67$ & 0.827 \\
\hline $\mathrm{T}_{3}, \mathrm{ng} / \mathrm{mL}(0.78-1.82)$ & $0.95 \pm 0.49$ & $1.52 \pm 0.10$ & 0.005 \\
\hline TPOAb positivity & $38 / 88(43.2)$ & $11 / 14(78.5)$ & $<0.001$ \\
\hline TgAb positivity & $22 / 88(25)$ & $8 / 14(57.1)$ & 0.068 \\
\hline \multicolumn{4}{|l|}{ Follow-up thyroid function tests } \\
\hline TSH, mIU/L (0.17-4.05) & $3.0 \pm 1.10$ & $18.8 \pm 21.99$ & $<0.001$ \\
\hline Free $\mathrm{T}_{4}, \mathrm{ng} / \mathrm{dL}(0.85-1.86)$ & $1.1 \pm 0.45$ & $0.79 \pm 0.48$ & 0.014 \\
\hline $\mathrm{T}_{3}, \mathrm{ng} / \mathrm{mL}(0.78-1.82)$ & $0.92 \pm 0.48$ & $1.03 \pm 0.43$ & 0.759 \\
\hline
\end{tabular}

Values are presented as mean $\pm \mathrm{SD}$, number (\%), or median (range).

TFT, thyroid function test; US, ultrasonography; TSH, thyroid stimulating hormone; T4, thyroxine; T3, triiodothyronine; TPOAb, thyroid peroxidase antibody; TgAb, thyroglobulin antibody.

${ }^{a}$ In this study, overt hypothyroidism and subclinical hypothyroidism patients were included as hypothyroidism group.

TFT every 3 to 6 months, but were found to remain in a subclinical hypothyroid state

\section{Factors that predict clinical hypothyroidism}

We evaluated potential clinical parameters at the time of FNA to find out predictable risk factors for future clinical hypothyroidism events (Table 4). In univariate analysis, the variables associated with clinical hypothyroidism were background thyroiditis on US (RR, 8.10; $p$ < 0.001 ; 95\% CI, 2.503 to 26.232), TPOAb positivity (RR, 7.34; $p=0.009 ; 95 \%$ CI, 1.640 to 32.844 ), initial TSH (RR , 1.55; $p<0.001 ; 95 \% \mathrm{CI}, 1.531$ to 1.580), nodule size (RR, 1.47; $p=0.004 ; 95 \% \mathrm{CI}, 1.415$ to 1.479$)$, and $\mathrm{TgAb}$ positivity (RR, 2.95; $p=0.054 ; 95 \% \mathrm{CI}, 1.640$ to 32.844$)$. In multivariate analysis, background thyroiditis on US (RR, 9.78; $p$ $=0.004 ; 95 \% \mathrm{CI}, 2.077$ to 46.124$)$, TPOAb positivity (RR, 9.90; $p=0.003 ; 95 \% \mathrm{CI}, 1.012$ to 96.887 ), nodule size (RR, 1.24; $p<0.001 ; 95 \%$ CI, 1.096 to 1.394), and initial TSH ( $R R, 1.47 ; p=0.009 ; 95 \% \mathrm{CI}, 1.011$ to 1.484 ) were found to be independent risk factors for predicting clinical hypothyroidism.

\section{DISCUSSION}

Thyroid nodules are detected frequently in clinical practice, and most of these thyroid nodules are benign. However, lymphocytic thyroiditis is the second most common thyroid lesion, after endemic goiter, and can lead to clinical hypothyroidism, which usually requires life-long thyroid hormone replacement [8]. Neverthe less, there are no definitive guidelines regarding follow-up TFT when FNAC reveals lymphocytic thyroiditis in a euthyroid patient. Thus, the present study evaluated the clinical outcomes of euthyroid patients with lymphocytic thyroiditis based on thyroid nodule cytology. Our results indicate that clinical hypothyroidism was reliably predicted by background thyroiditis (RR, 9.78) 
Table 3. Initial clinical characteristics of patients with overt hypothyroidism as final outcome

\begin{tabular}{|c|c|c|c|c|c|c|}
\hline Patient no. & Age, yr & Sex & TPOAb positivity & Nodule size, mm & $\begin{array}{l}\text { Background thyroiditis } \\
\text { grading }(1-4)^{\mathrm{a}}\end{array}$ & Initial TSH, mIU/L \\
\hline 1 & 60 & $\mathrm{~F}$ & - & 35.0 & 3 & 3.67 \\
\hline 2 & 30 & $\mathrm{~F}$ & + & 10.2 & 2 & 1.57 \\
\hline 3 & 62 & $\mathrm{~F}$ & + & 36.1 & 4 & 4.04 \\
\hline 4 & 28 & $\mathrm{~F}$ & + & 29.0 & 4 & 2.31 \\
\hline 5 & 59 & $\mathrm{~F}$ & + & 60.0 & 3 & 2.58 \\
\hline 6 & 43 & M & + & 21.0 & 4 & 0.18 \\
\hline 7 & 50 & $\mathrm{~F}$ & + & 31.0 & 2 & 3.81 \\
\hline 8 & 56 & $\mathrm{~F}$ & + & 26.0 & 4 & $3 \cdot 75$ \\
\hline 9 & 34 & $\mathrm{~F}$ & - & 29.0 & 4 & 4.04 \\
\hline 10 & 51 & $\mathrm{~F}$ & + & 22.0 & 4 & 0.17 \\
\hline 11 & 44 & $\mathrm{~F}$ & - & 16.0 & 4 & 1.17 \\
\hline 12 & 47 & M & + & 22.0 & 2 & 4.04 \\
\hline 13 & 36 & $\mathrm{~F}$ & + & 24.0 & 4 & 4.03 \\
\hline 14 & 67 & $\mathrm{~F}$ & + & 24.0 & 2 & 2.20 \\
\hline
\end{tabular}

TPOAb, thyroid peroxidase antibody; TSH, thyroid stimulating hormone.

${ }^{a}$ Background thyroiditis grading is determined based on suggestion of Sostre and Reyes. Grade 1 indicates only enlarged gland with a normoechoic pattern; Grade 2 presents as multiple hypoechogenic foci throghout normoechoic gland; Grade 3 indicates enlarged gland with diffuse hypoechogenicity; Grade 4 indicates severe thyroiditis with thyroid enlargement and marked multiple hypoechogenicity.

Table 4. Univariate and multivariate analysis for the development of hypothyroidism (Cox models)

\begin{tabular}{|c|c|c|c|c|c|c|}
\hline \multirow{2}{*}{ Variable } & \multicolumn{3}{|c|}{ Univariate (unadjusted) analysis } & \multicolumn{3}{|c|}{ Multivariate (adjusted) analysis } \\
\hline & Relative risk & $95 \% \mathrm{CI}$ & $p$ value & Relative risk & $95 \% \mathrm{CI}$ & $p$ value \\
\hline Age & 0.98 & $0.943-1.026$ & 0.451 & & & \\
\hline Female sex & 1.67 & $0.235-4.854$ & 0.547 & & & \\
\hline Nodule size, mm & 1.47 & $1.415^{-1.479}$ & 0.004 & 1.24 & $1.096-1.394$ & $<0.001$ \\
\hline $\mathrm{US}\left(\mathrm{G}_{1}-\mathrm{G}_{2} / \mathrm{G}_{3}-\mathrm{G}_{4}\right)$ & 8.10 & $2.503-26.232$ & $<0.001$ & 9.78 & $2.077-46.124$ & 0.004 \\
\hline TSH, mIU/L & 1.55 & $1.531-1.580$ & $<0.001$ & 1.47 & $1.011-1.484$ & 0.009 \\
\hline Initial free $\mathrm{T}_{4}, \mathrm{ng} / \mathrm{dL}$ & 0.21 & $0.072-1.63$ & 0.727 & 0.13 & $0.013-1.277$ & 0.083 \\
\hline Initial $\mathrm{T}_{3}, \mathrm{ng} / \mathrm{mL}$ & 1.39 & $0.884-2.184$ & 0.153 & 1.59 & $0.597-4.227$ & 0.354 \\
\hline TPOAb positivity & $7 \cdot 34$ & $1.640-32.844$ & 0.009 & 9.90 & $1.012-96.887$ & 0.003 \\
\hline TgAb positivity & 2.95 & $0.980-8.904$ & 0.054 & 2.88 & $0.912-9.061$ & 0.071 \\
\hline
\end{tabular}

CI, confidence interval; US, ultrasonography; TSH, thyroid stimulating hormone; T4, thyroxine; T3, triiodothyronine; TPOAb, thyroid peroxidase antibody; $\mathrm{TgAb}$, thyroglobulin antibody.

and TPOAb positivity ( $R R, 9.90)$.

The severity of background thyroiditis during US was a strong predictor of clinical hypothyroid events. For example, $88.6 \%$ of the patients in group E had grade 1 to 2 background thyroiditis during US, while $71.4 \%$ of the patients in group $\mathrm{H}$ had grade 3 to 4 background thy- roiditis. According to Pedersen et al. [15], inflammation is presented as marked hypoechogenicity because of thyroid parenchymal infiltration with lymphocytes and fibrosis [12]. Decreased echogenicity or irregular heterogeneous echo patterns are also positive findings for thyroiditis [16]. Hypoechogenicity has been suggested as an 
early sign of thyroid failure [17].

Moreover, one study [18] of 482 healthy subjects revealed that 41 individuals (8.5\%) exhibited mild-tomarked hypoechogenicity and background thyroiditis during thyroid US, with 11 of these individuals (26.8\%) developing clinical hypothyroidism.

In group $\mathrm{H}$, the dominant nodule was approximately 2-fold larger than that in group E (27.5 mm vs. $15.1 \mathrm{~mm})$. In this context, previous research has indicated that nodule size may be influenced by TSH stimulation in patients with a solitary thyroid nodule [19]. However, the present study failed to detect a significant correlation between nodule size and TSH levels (data not shown). Nevertheless, marked hypoechogenicity in lymphocytic thyroiditis is attributed to thyroid lymphocyte infiltration [20] and echogenic diversity based on the amount of fibrosis may determine the nodularity and nodule size [21]. Therefore, hypoechogenicity and background thyroiditis are possibly correlated with initial nodule size, which might indicate the extent of local thyroiditis. All $\mathrm{G}_{3} / 4$ patients in the $\mathrm{H}$ group had larger nodules than $\mathrm{Gr} / 2$ patients in the $\mathrm{H}$ group $(27.9 \pm 12.0 \mathrm{~mm}$ vs. $17.7 \pm$ $8.7 \mathrm{~mm})$.

TPOAb positivity was a significant risk factor in the univariate analysis and a strong correlation was observed in group $\mathrm{H}$. In this context, detectable TPOAb titers precede elevated TSH and decreased free T4 levels, although TPOAb can be detected in euthyroid patients [22]. Several studies had revealed that high TPOAb titers are related to the severity of lymphocytic infiltration, despite the absence of clinical hypothyroidism [23,24]. A prospective Dutch study also revealed that 206 of 739 euthyroid patients (28\%) exhibited TPOAb positivity, and 38 of the patients with TPOAb positivity (5.1\%) developed overt hypothyroidism during a 5-year follow-up, although the report did not describe the time to onset of clinical hypothyroidism. Moreover, a 20-year follow-up Whickham survey [25] of the general population's incidence of thyroid antibodies revealed that 313 of 1,704 individuals (18.4\%) exhibited TPOAb positivity, 81 of 1,017 individuals $(8.0 \%)$ were diagnosed with clinical hypothyroidism, and 16 of the 81 individuals developed hypothyroidism within 1 year.

In addition to TPOAb, TgAb is associated with a histological diagnosis of autoimmune thyroiditis and the development of clinical hypothyroidism. However, the present study indicates that TgAb was not a significant risk factor for hypothyroidism. In addition, previous reports have indicated that $\mathrm{TgAb}$ alone is not strongly correlated with overt hypothyroidism $[26,27]$.

TSH levels can predict the progression from a euthyroid status to overt hypothyroidism $[25,28,29]$. Similarly, the Whickham survey [25] suggested that an initial TSH level of $>2.0 \mathrm{mIU} / \mathrm{L}$ was an independent risk factor for future clinical hypothyroidism.

In our study, the median onset time to clinical hypothyroidism was 16 months. This time point appears to be closer than expected. Because almost all patients of the $\mathrm{H}$ group had a severity corresponding to US findings and TPOAb positivity at first visit, they were detected at short follow-up. Thus, clinicians should check TFT every 6 months within 2 years in high risk patients.

The present study aimed to develop an algorithm (Fig. 2) for using appropriate follow-up tools and identifying risk factors for clinical hypothyroidism in cases with a euthyroid status but lymphocytic thyroiditis identified by FNAC. The first step is that patients with confirmed lymphocytic thyroiditis on FNAC and grade 3 to 4 thyroid US findings should undergo TFT within 6 months. Cases with grade 1 to 2 thyroid US findings should be evaluated for TPOAb positivity, which indicates the need for follow-up TFT. Patients without TPOAb should undergo an evaluation of the nodule's size, and cases with large nodules $(>17 \mathrm{~mm}$ ) should be regularly evaluated using TFT, although this approach is not necessary for patients with a small nodule $(<17 \mathrm{~mm})$ and TSH levels of $<2.02 \mathrm{mIU} / \mathrm{L}$.

Applying to algorithm of Fig. 2, 20 patients whose thyroid US finding was grade 3 or 4 should undergo regular follow-up from the beginning. Among 89 patients whose US finding was appropriate to grade 1 or 2, 30 of them who had TPOAb positivity, which is the second most common risk factor, should check TFT regularly. Sixteen with nodule size greater than $17 \mathrm{~mm}$ should be considered regular follow-up in the remaining 59 patients with TPOAb negativity. Whereas 32 patients with less than $17 \mathrm{~mm}$ sized nodules and low TSH levels did not need further follow-up.

The present study has several limitations. The first limitation is the retrospective design, which is associated with known risks of bias. For example, more hypothyroid events occurred under close observation within 


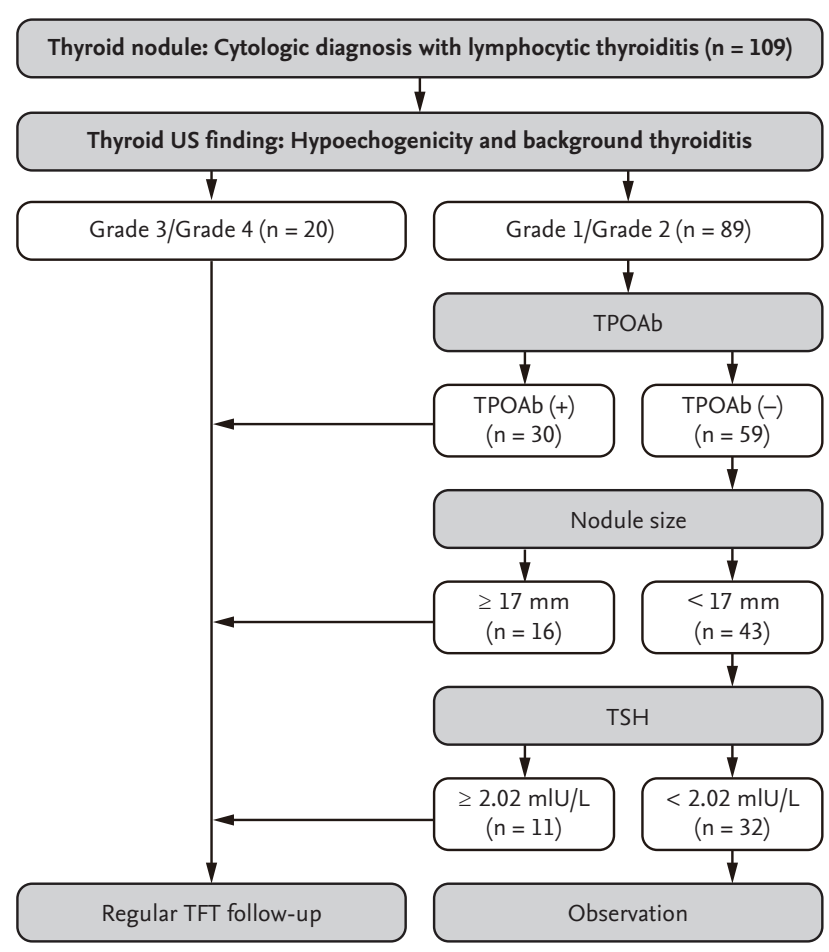

Figure 2. Algorithm for follow-up of thyroid nodule with Hashimoto's thyroiditis. Patients with a thyroid nodule and Hashimoto's thyroiditis, based on fine needle aspiration cytology, should be evaluated to identify risk factors for hypothyroidism. Follow-up thyroid function test (TFT) is indicated at $<6$ months if the patient has a high thyroid ultrasonography grade, is positive for antibodies to thyroid peroxidase, has a large nodule, or has elevated thyroid stimualting hormone levels. Observation is sufficient for patients with lower grade thyroid ultrasonography findings, no antibodies to thyroid peroxidase, a nodule that has a diameter of $<1.7 \mathrm{~cm}$, or thyroid-stimulated hormone levels of $<2.02 \mathrm{mIU} / \mathrm{L}$. US, ultrasonographic; TPOAb, thyroid peroxidase antibody; TSH, thyroid stimulating hormone.

1 year of follow-up, and fewer events occurred at $>1$ year with increasing losses to follow-up. Nevertheless, $78.6 \%$ of the patients in group $\mathrm{H}$ (11/14) progressed to hypothyroidism within 1 year, while progression in group $\mathrm{E}$ generally required $>1$ year. The second limitation is the small sample size, which is likely related to the limited number of euthyroid patients who are confirmed to have lymphocytic thyroiditis on FNAC. In general, TFT is not included in the follow-up of nodules without lymphocytic thyroiditis that are detected on FNAC. Therefore, in this study, only patients with cytologically diagnosed lymphocytic thyroiditis were enrolled and problems were found in the control group setting. There was no data comparing TFT follow-up with non-lymphocytic thyroiditis group in our study.

In conclusion, the existing clinical guidelines indicate that benign nodules confirmed on FNAC can be followed using US, although patients with an initial euthyroid state at the time of the FNAC rarely undergo repeated TFT [30-32]. However, some patients with lymphocytic thyroiditis on FNAC progress to clinical hypothyroidism. These patients are likely to exhibit hypoechogenic background thyroiditis, TPOAb positivity, large nodules, and high TSH levels. Thus, regular TFT is needed in these cases to identify the progression to clinical hypothyroidism, and further prospective research is required to confirm whether our proposed algorithm can be implemented in clinical practice.

\section{KEY MESSAGE}

1. Lymphocytic thyroiditis is the second most common lesion diagnosed by fine needle aspiration cytology (FNAC) and can progress to clinical hypothyroidism.

2. In euthyroid patients with lymphocytic thyroiditis on FNAC, clinical hypothyroidism could be predicted by background thyroiditis on ultrasonography, thyroid peroxidase antibody positivity, nodule size, and thyroid stimulating hormone levels.

3. Clinician should check thyroid function test regularly in patients with lymphocytic thyroiditis on FNAC.

\section{Conflict of interest}

No potential conflict of interest relevant to this article was reported.

\section{Acknowledgments}

A portion of this study was presented in abstract form at the Endocrine Society's 2016 meeting.

\section{REFERENCES}

1. McDougall IR. Epidemiology and etiology of thyroid 
nodules and thyroid cancers. In: McDougall IR, ed. Thyroid Cancer in Clinical Practice. London: Springer, 2007:1-16.

2. Burch HB, Burman KD, Cooper DS, Hennessey JV, Vietor NO. A 2015 survey of clinical practice patterns in the management of thyroid nodules. J Clin Endocrinol Metab 2016;101:2853-2862.

3. Mortensen JD, Woolner LB, Bennett WA. Gross and microscopic findings in clinically normal thyroid glands. J Clin Endocrinol Metab 1955;15:1270-1280.

4. Gayathri B, Kalyani R, Harendra KM, Krishna PK. Fine needle aspiration cytology of Hashimoto's thyroiditis: a diagnostic pitfall with review of literature. J Cytol 2011;28:210-213.

5. Surks MI, Ortiz E, Daniels GH, et al. Subclinical thyroid disease: scientific review and guidelines for diagnosis and management. JAMA 2004;291:228-238.

6. Ross DS. Serum thyroid-stimulating hormone measurement for assessment of thyroid function and disease. Endocrinol Metab Clin North Am 2001;30:245-264.

7. Parle JV, Franklyn JA, Cross KW, Jones SC, Sheppard MC. Prevalence and follow-up of abnormal thyrotrophin (TSH) concentrations in the elderly in the United Kingdom. Clin Endocrinol (Oxf) 1991;34:77-83.

8. Kumar N, Ray C, Jain S. Aspiration cytology of Hashimoto's thyroiditis in an endemic area. Cytopathology 2002;13:3139.

9. Guaraldi F, Zang G, Dackiw AP, Caturegli P. Oncocytic mania: a review of oncocytic lesions throughout the body. J Endocrinol Invest 2011;34:383-394.

10. Lim DJ, Kim JY, Baek KH, et al. Natural course of cytologically benign thyroid nodules: observation of ultrasonographic changes. Endocrinol Metab (Seoul) 2013;28:110118.

11. Sostre S, Reyes MM. Sonographic diagnosis and grading of Hashimoto's thyroiditis. J Endocrinol Invest 1991;14:115-121.

12. Marcocci C, Vitti P, Cetani F, Catalano F, Concetti R, Pinchera A. Thyroid ultrasonography helps to identify patients with diffuse lymphocytic thyroiditis who are prone to develop hypothyroidism. J Clin Endocrinol Metab 1991;72:209-213.

13. Sarikaya B, Demirbilek H, Akata D, Kandemir N. The role of the resistive index in Hashimoto's thyroiditis: a sonographic pilot study in children. Clinics (Sao Paulo) 2012;67:1253-1257.
14. Park JW, Kim DW, Kim D, Baek JW, Lee YJ, Baek HJ. Korean Thyroid Imaging Reporting and Data System features of follicular thyroid adenoma and carcinoma: a single-center study. Ultrasonography 2017;36:349-354.

15. Pedersen OM, Aardal NP, Larssen TB, Varhaug JE, Myking $\mathrm{O}$, Vik-Mo H. The value of ultrasonography in predicting autoimmune thyroid disease. Thyroid 2000;10:251-259.

16. Kim I, Kim EK, Yoon JH, et al. Diagnostic role of conventional ultrasonography and shearwave elastography in asymptomatic patients with diffuse thyroid disease: initial experience with 57 patients. Yonsei Med J 2014;55:247-253.

17. Rhee SJ, Hong HS, Kim CH, Lee EH, Cha JG, Jeong SH. Using acoustic structure quantification during b-mode sonography for evaluation of Hashimoto thyroiditis. J Ultrasound Med 2015;34:2237-2243.

18. Rago T, Chiovato L, Grasso L, Pinchera A, Vitti P. Thyroid ultrasonography as a tool for detecting thyroid autoimmune diseases and predicting thyroid dsfunction in apparently healthy subjects. J Endocrinol Invest 2001;24:763769 .

19. Bennedbaek FN, Perrild H, Hegedus L. Diagnosis and treatment of the solitary thyroid nodule. Results of a European survey. Clin Endocrinol (Oxf) 1999;50:357-363.

20. Yeh HC, Futterweit W, Gilbert P. Micronodulation: ultrasonographic sign of Hashimoto thyroiditis. J Ultrasound Med 1996;15:813-819.

21. Reading CC, Charboneau JW, Hay ID, Sebo TJ. Sonography of thyroid nodules: a "classic pattern" diagnostic approach. Ultrasound Q 2005;21:157-165.

22. Mcconahey WM, Keating FR jr, Butt HR, Owen CA jr. Comparison of certain laboratory tests in the diagnosis of Hashimoto's thyroiditis. J Clin Endocrinol Metab 1961;21:879-886.

23. Kasagi K, Kousaka T, Higuchi K, et al. Clinical significance of measurements of antithyroid antibodies in the diagnosis of Hashimoto's thyroiditis: comparison with histological findings. Thyroid 1996;6:445-450.

24. Li Y, Teng D, Shan Z, et al. Antithyroperoxidase and antithyroglobulin antibodies in a five-year follow-up survey of populations with different iodine intakes. J Clin Endocrinol Metab 2008;93:1751-1757.

25. Vanderpump MP, Tunbridge WM, French JM, et al. The incidence of thyroid disorders in the community: a twenty-year follow-up of the Whickham Survey. Clin Endocri$\operatorname{nol}($ Oxf) 1995;43:55-68.

26. Hollowell JG, Staehling NW, Flanders WD, et al. Serum 
TSH, T(4), and thyroid antibodies in the United States population (1988 to 1994): National Health and Nutrition Examination Survey (NHANES III). J Clin Endocrinol Metab 2002;87:489-499.

27. O'Leary PC, Feddema PH, Michelangeli VP, et al. Investigations of thyroid hormones and antibodies based on a community health survey: the Busselton thyroid study. Clin Endocrinol (Oxf) 2006;64:97-104.

28. Strieder TG, Tijssen JG, Wenzel BE, Endert E, Wiersinga WM. Prediction of progression to overt hypothyroidism or hyperthyroidism in female relatives of patients with autoimmune thyroid disease using the Thyroid Events Amsterdam (THEA) score. Arch Intern Med 2008;168:16571663.

29. Lee MW, Shin DY, Kim KJ, Hwang S, Lee EJ. The bio- chemical prognostic factors of subclinical hypothyroidism. Endocrinol Metab (Seoul) 2014;29:154-162.

30. Haugen BR, Alexander EK, Bible KC, et al. 2015 American Thyroid Association management guidelines for adult patients with thyroid nodules and differentiated thyroid cancer: the American thyroid Association guidelines task force on thyroid nodules and differentiated thyroid cancer. Thyroid 2016;26:1-133.

31. Hennessey JV, Espaillat R. Subclinical hypothyroidism: a historical view and shifting prevalence. Int J Clin Pract 2015;69:771-782.

32. Amino N, Miyai K, Fukuchi M, Kumahara Y. Transient hypothyroidism associated with increased anti-microsomal antibodies. Endocrinol Jpn 1975;22:141-146. 


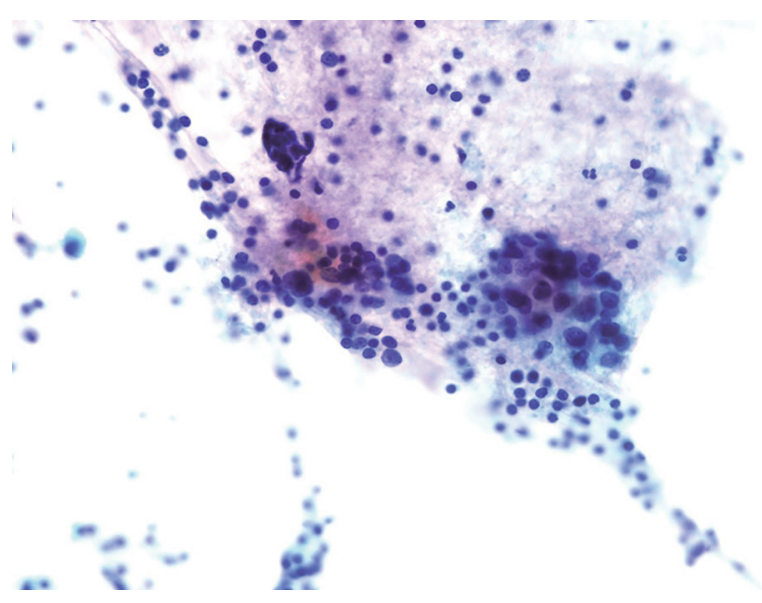

Supplementary Figure 1. Cytologic finding of lymphocytic thyroiditis. The progressive loss of follicular cells and their concomitant replacement by a lymphoid infiltrate is visible (Papanicolaou stain, $\times 400$ ). 